\title{
P201: First report in the world of Mycobacterium bacteremicum causing a cluster of postlaparotomy surgical wound infections
}

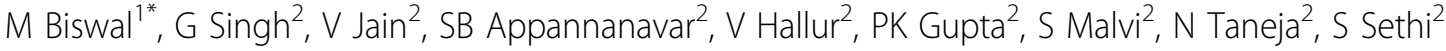 \\ From 2nd International Conference on Prevention and Infection Control (ICPIC 2013) \\ Geneva, Switzerland. 25-28 June 2013
}

\section{Introduction}

Uncommon atypical mycobacteria previously known to be environmental contaminants are an increasingly reported cause of outbreaks of surgical site wound infections. We investigated a cluster of post-laparotomy wound infections in 12 patients at our tertiary care hospital in India. We describe the epidemiology and the methods used to investigate the outbreak.

\section{Objectives}

The objective of this study was to investigate a cluster of post-laparotomy wound infections in 12 patients at our tertiary care hospital in India using 16SrRNA typing.

\section{Methods}

The outbreak started in October, 2011 and continued till April, 2012. The patients presented with delayed wound healing post laparotomy surgery. Swabs collected from the gaping wounds were sent for culture of atypical mycobacteria. Samples were also collected from the environment to locate the source of the organism. Samples were plated on Middlebrook 7H10 and LowensteinJensen medium. Mycobacteria were identified by partial 16S r RNA sequencing.

\section{Results}

All specimens yielded a yellow pigmented rapidly growing mycobacterium species. The sequences (Seq1 and Seq2) obtained by PCR using $16 \mathrm{~S}$ rRNA PCR were compared with that in the GenBank database. The sequences of our isolates gave $99 \%$ identity with the ex-type strain of Mycobacterium bacteremicum (ATCC 25791).
Sequence alignment and phylogenetic tree were constructed using the neighbour-joining method with MEGA5.1software package. Sequence data were submitted to the GenBank (Accession No. JX473587 \& JX473588).

\section{Conclusion}

In conclusion, delayed wound healing in surgical patients should be investigated for atypical mycobacteria using molecular methods to reach a diagnosis and institute appropriate and prolonged antimicrobial treatment. To the best of our knowledge, the causative agent, $M$. bacteremicum is being reported to cause post-surgical wound infection for the first time in world literature.

\section{Disclosure of interest}

None declared

Author details

${ }^{1}$ Department of Medical Microbiology, Chandigarh, India. ${ }^{2}$ Postgraduate Institute of Medical Education and Research, Chandigarh, India.

Published: 20 June 2013

doi:10.1186/2047-2994-2-S1-P201

Cite this article as: Biswal et al:: P201: First report in the world of Mycobacterium bacteremicum causing a cluster of postlaparotomy surgical wound infections. Antimicrobial Resistance and Infection Control 2013 2(Suppl 1):P201.

${ }^{1}$ Department of Medical Microbiology, Chandigarh, India

Full list of author information is available at the end of the article

(c) 2013 Biswal et al; licensee BioMed Central Ltd. This is an Open Access article distributed under the terms of the Creative Commons 